\title{
Laterally self-ordered silicon-germanium islands with optimized confinement properties
}

\author{
Thomas Zabel, ${ }^{1,2, a)}$ Narayan Sircar, ${ }^{1,2}$ Norman Hauke, ${ }^{1}$ Josef Zweck, ${ }^{2}$ Markus Döblinger, ${ }^{3}$ \\ Michael Kaniber, ${ }^{1}$ Jonathan J. Finley, ${ }^{1}$ Gerhard Abstreiter, ${ }^{1,4}$ Yasuhiko Arakawa, ${ }^{4,5}$ \\ and Dominique Bougeard ${ }^{2}$ \\ ${ }^{1}$ Walter Schottky Institut and Physik Department, Technische Universität München, Am Coulombwall 4, \\ D-85748 Garching, Germany \\ ${ }^{2}$ Institut für Experimentelle und Angewandte Physik, Universität Regensburg, D-93040 Regensburg, Germany \\ ${ }^{3}$ Department of Chemistry, Ludwig Maximilian Universität München, D-81377 Munich, Germany \\ ${ }^{4}$ Institute for Advanced Study, Technische Universität München, D-85748 Garching, Germany \\ ${ }^{5}$ Institute of Industrial Science, Institute for Nano Quantum Information Electronics, The University of Tokyo, \\ 4-6-1 Komaba, Meguro-ku, Tokyo 153-8505, Japan
}

(Received 11 May 2013; accepted 27 July 2013; published online 8 August 2013)

\begin{abstract}
We present an analysis of the electronic confinement properties of self-assembled islands forming via silicon and germanium co-deposition in molecular beam epitaxy. This approach allows the fabrication of laterally self-ordered three dimensional islands in the Stranski-Krastanow growth mode. Using a systematic structural analysis, we derive a realistic fit-parameter free island model for band structure simulations. A comparison between these band structure simulations and photoluminescence spectroscopy shows that such islands have a significant three dimensional spatial electron-hole wave function overlap. In addition, we show that this spatial wave function overlap overcompensates a weak wave function spreading in k-space. (C) 2013 AIP Publishing LLC. [http://dx.doi.org/10.1063/1.4818331]
\end{abstract}

Optically integrated circuits ${ }^{1}$ present a promising platform for high bandwidths and fast data transmission speed in information processing. To interconvert electrical and optical signals, an on-chip integration of light sources into the well established silicon ( $\mathrm{Si}$ ) electronics technology is highly desirable. Since bulk $\mathrm{Si}$ is a rather poor light emitter, due to its indirect band gap, ${ }^{2}$ several approaches are currently pursued regarding the realization of on-Si-chip light sources. Recently, progress has been made in a hybrid approach where III-V light emitting stacks are integrated in Si-chips with high radiative efficiency ${ }^{3}$ and bandwidth. ${ }^{4}$ An alternative route, allowing a monolithic integration on $\mathrm{Si}$, could be based on the introduction of suitable nanostructures as emitters. In nanostructures, the spatial confinement opens possibilities to tailor the spatial overlap of charge carrier wave functions in real space on the one hand and to induce a spreading of the wave functions in $\mathrm{k}$-space on the other hand. Examples of tested nanostructures include Erbium doping, 5 anodized porous silicon ${ }^{6,7}$ or low dimensional silicon-germanium ( $\mathrm{SiGe}$ ) heterostructures. ${ }^{8-11}$ For the latter, the efficiency of the carrier confinement is determined by the distribution of the band offset between conduction and valence bands ${ }^{12,13}$ and the spatial dimensions of the SiGe heterostructures..$^{8-11}$ Ideally, an optimization of both is desired.

This letter addresses this optimization by exploring a co-deposition approach of self-assembled SiGe islands. We observe the formation of islands in the Stranski-Krastanow growth mode for nominal layer thicknesses larger than 25 monolayers (ML). For certain island ensembles we, furthermore, find a pronounced lateral self-ordering along the $\{100\}$ direction without pre- or post processing steps. These islands exhibit two equally strong radiative recombination

a)zabel@wsi.tum.de channels, one being indirect and one being direct in k-space. Both have a remarkably high intensity compared to the matrix material at $10 \mathrm{~K}$. The occurrence of a phonon transition allows to deduce a rather weak wave function spreading in $\mathrm{k}$-space. A comparison of photoluminescence (PL) measurements with $\mathrm{k} \cdot \mathrm{p}$ band structure simulations based on a realistic island model indicates a band offset resulting in a spatial confinement of both electrons and holes within the SiGe islands. This confinement situation is typically not observed in Ge based islands on $\mathrm{Si}^{14}$ and allows a larger spatial overlap of the electron and hole wave functions. The resulting larger oscillator strength seems to overcompensate the weak wave function spreading in $\mathrm{k}$-space in our SiGe islands.

All samples have been grown using standard Si (001) wafers. Growth was initialized with an $80 \mathrm{~nm}$ Si buffer layer which results in an atomically flat surface. A film equivalent nominal thickness of $20-80 \mathrm{ML} \mathrm{Si}_{0.7} \mathrm{Ge}_{0.3}$ was deposited at a wafer temperature of $630{ }^{\circ} \mathrm{C}$ and a combined growth rate of $0.58 \mathrm{ML} / \mathrm{s}$. Si and Ge were co-evaporated using two separate electron beam evaporator cells. The islands have been capped with $\mathrm{Si}$ at $630^{\circ} \mathrm{C}$. Finally, an uncapped layer of $\mathrm{Si}_{0.7} \mathrm{Ge}_{0.3}$ was deposited using the same growth conditions as the optically active layer for topographic island analysis.

Atomic force microscopy (AFM) and cross-sectional transmission electron microscopy (TEM) including energy dispersive X-ray spectroscopy (EDX) and Raman spectroscopy $^{15}$ was used for structural analysis of the island ensembles. Optical interband PL experiments at 10 and $20 \mathrm{~K}$ have been conducted using the $488 \mathrm{~nm}$ line of an argon ion laser. The excitation power density was approximately $40 \mathrm{Wcm}^{-2}$. The emission signal was dispersed by a grating monochromator and detected using a liquid nitrogen cooled Ge detector in lock-in technique. Realistic three-dimensional eight-band $\mathrm{k} \cdot \mathrm{p}$ band structure calculations were performed 
using nextnano.$++{ }^{16}$ The $\Delta$-band was added solving the single band Schrödinger equation.

Figs. 1(a)-1(c) show AFM images of samples grown with nominal $\mathrm{Si}_{0.7} \mathrm{Ge}_{0.3}$ thicknesses of 20, 35, and $80 \mathrm{ML}$. The insets include the 2D fast Fourier transformed (FFT) images of the corresponding AFM scans. AFM measurements performed on the sample grown with nominally 20 ML reveal a flat surface. The TEM analysis (not shown here) indicates that the capped $20 \mathrm{ML}$ thick $\mathrm{Si}_{0.7} \mathrm{Ge}_{0.3}$ layer forms a dislocation-free pseudomorphically strained quantum well. Upon the deposition of nominally 25 to $55 \mathrm{ML}$ of $\mathrm{Si}_{0.7} \mathrm{Ge}_{0.3}$, the surface is covered by pyramids with a quadratic base (mainly $\{105\}$ faceted). Thus, we conclude that the critical thickness of a $\mathrm{Si}_{0.7} \mathrm{Ge}_{0.3}$ alloy grown on $\mathrm{Si}$ is between 20-25 ML. Typical island ensembles are exemplarily shown in Figs. 1(b) and 1(c) for nominally $35 \mathrm{ML}$ and $80 \mathrm{ML}$ of $\mathrm{Si}_{0.7} \mathrm{Ge}_{0.3}$. For thicknesses higher than $55 \mathrm{ML}$, the pyramidal shape of the islands vanishes in favor of more round, dome-like islands.

Interestingly, we observe that between a nominal deposition of 30 to $55 \mathrm{ML}$, the quadratically based pyramids very regularly self-order along the densest possible pattern, rectangularly along $\{100\}$ as indicated with white rectangles in the left inset of Fig. 1(b). This is also reflected in the clear four fold symmetry of the corresponding Fourier transform images. For nominal $\mathrm{Si}_{0.7} \mathrm{Ge}_{0.3}$ thicknesses higher than $55 \mathrm{ML}$, as the pyramidal shape of the islands vanishes, the degree of ordering reduces, as can exemplarily be seen for $80 \mathrm{ML}$ in Fig. 1(c). Here, some islands begin to occupy interstitial lattice sites of the ordered lattice. The TEM analysis reveals that the lateral ordering is also maintained during the capping process. Further studies on this self-ordering mechanism of the pyramidal island ensemble might be interesting for optical and electrical applications which require the exact

(a)

(c)

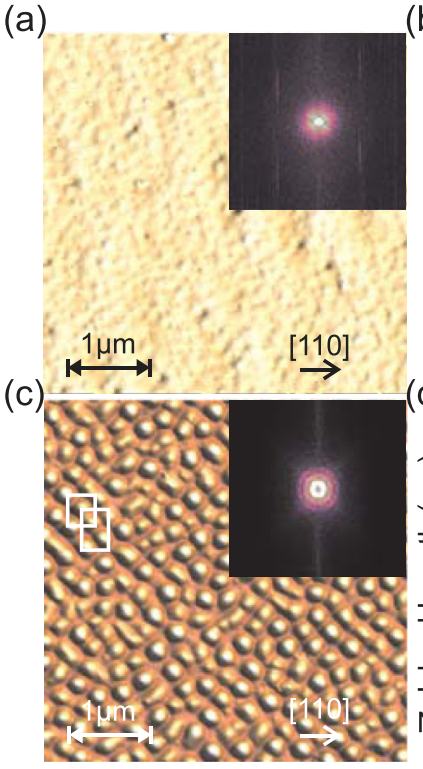

(b)

(d)
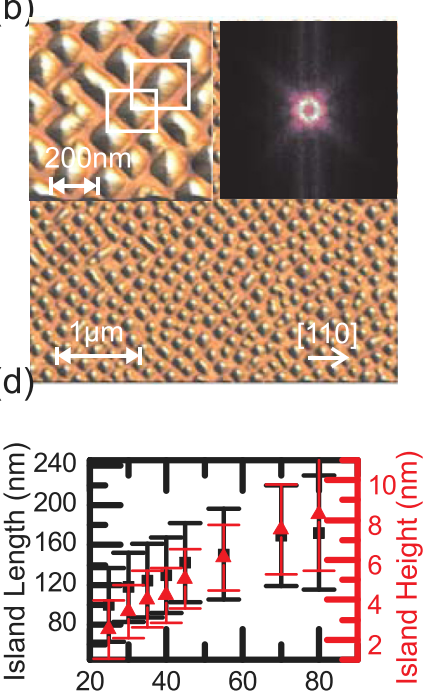

FIG. 1. Atomic force microscopy images of the $\mathrm{Si}_{0.7} \mathrm{Ge}_{0.3}$ surface after deposition of nominally (a) $20 \mathrm{ML}$, (b) $35 \mathrm{ML}$ (the left inset shows a zoom) and (c) $80 \mathrm{ML} \mathrm{Si}_{0.7} \mathrm{Ge}_{0.3}$ on $\mathrm{Si}$ at $630^{\circ} \mathrm{C}$. The right inset shows the corresponding $2 \mathrm{D}$ fast Fourier transformed image. The white rectangles indicate the ordered pattern along the $\{100\}$ direction. (d) Dependence of the island length (black squares) and the island height (red triangles) as a function of deposited monolayers. positioning of cavities or contacts relative to the islands, as here no pre- or post processing of the island ensemble is required. ${ }^{17}$

In Fig. 1(d), both the island base length (squares) and height (triangles) determined from AFM are depicted as a function of the amount of nominally deposited monolayers for all studied samples. The statistical size variation of the islands results in the large error bars. The TEM study indicates that up to a nominal thickness of $80 \mathrm{ML} \mathrm{Si}_{0.7} \mathrm{Ge}_{0.3}$ the islands are dislocation-free and that the island shape is maintained during capping.

We use the information deduced from structural analysis to define a realistic island model. The island dimensions and shape are obtained from AFM and TEM. The average Ge content is determined by Raman spectroscopy. Spatially resolved EDX scans revealed a linear Ge profile with a Ge rich apex and a Si rich base. The wetting layer thickness is calculated from the difference of the total volume of the deposited material and the island volume. In order to verify the validity of the chosen island model, we compare band structure simulations with systematic PL measurements. Exemplarily, we present, in Fig. 2(a), the normalized PL spectrum of a $\mathrm{Si}_{0.7} \mathrm{Ge}_{0.3}$ island ensemble, consisting of nominally $40 \mathrm{ML} \mathrm{Si}_{0.7} \mathrm{Ge}_{0.3}$. From $1 \mathrm{eV}$ to $1.14 \mathrm{eV}$, we find the $\mathrm{Si}-\mathrm{TO}+\mathrm{O}^{\Gamma}$, the $\mathrm{Si}-\mathrm{TO}$ and the Si-TA recombination lines, stemming from the $\mathrm{Si}$ matrix and their corresponding
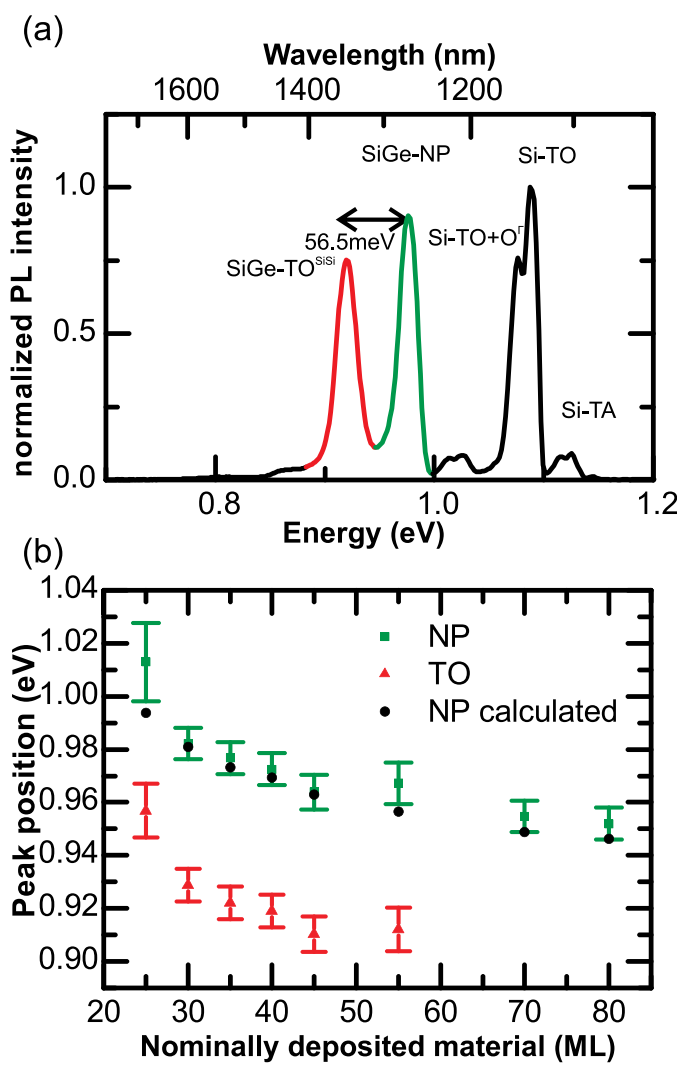

FIG. 2. (a) Photoluminescence spectrum of an ordered ensemble of $\mathrm{Si}_{0.7} \mathrm{Ge}_{0.3}$ islands with a nominal deposition of $40 \mathrm{ML}$ upon excitation of $4 \mathrm{~mW}$ at a wavelength of $488 \mathrm{~nm}$ and a temperature of $10 \mathrm{~K}$. (b) Dependence of the experimental no-phonon (green squares) and SiGe transversal optical phonon (red triangles) from the island ensemble as well as the calculated nophonon (black circles) peak positions as a function of the deposited material. The measurements have been performed at $18 \mathrm{~K}$ and an excitation power of $2.3 \mathrm{~mW}$ 
electron hole droplets. ${ }^{18,19}$ In addition, the $\mathrm{Si}_{0.7} \mathrm{Ge}_{0.3}$ islands exhibit a luminescence peak at $\approx 0.976 \mathrm{eV}$ and a second peak at $0.920 \mathrm{eV}$. The energy difference between these two peaks equals $56.5 \mathrm{meV}$ which is characteristic for the energy of a $\mathrm{SiGe}$ transversal optical phonon in a Si lattice $\left(\mathrm{SiGe}-\mathrm{TO}^{\text {SiSi }}\right)$. Hence, we attribute the peak at $\approx 0.976 \mathrm{eV}$ to a no-phonon recombination in the islands. The second peak stems from the $\mathrm{Si}_{0.7} \mathrm{Ge}_{0.3}$ island $\mathrm{SiGe}-\mathrm{TO}^{\text {SiSi }}$ phonon replica. ${ }^{20}$

Fig. 2(b) summarizes the experimentally determined transition energies of the no-phonon (squares) and TOphonon (triangles) transitions for all studied samples. Both transitions red-shift as a function of the nominally deposited amount of material. We also plot the transition energies of each investigated island ensemble as calculated in our 3D band structure simulations (circles). The agreement between experimental and simulation data is excellent. We would like to strengthen that the simulation uses only experimentally determined structural input parameters for the island model. No fitting parameter was used as an input for the comparison with the PL data, assessing the validity of our model. With increasing material deposition the island height increases, resulting in a shift of the energy states closer to the band edge of the $\mathrm{Si}_{0.7} \mathrm{Ge}_{0.3}$ alloy. Thus, the red-shift is a clear evidence for the presence of a quantum confinement effect.

Moreover, we note that the intensity of the phononassisted recombination is of the same order of magnitude than the no-phonon transition. This might seem surprising, as phonon-assisted three-particle processes are less probable than no-phonon two-particle transitions. As no-phonon transitions require k-vector conservation, the observed experimental behavior suggests that only few of the recombining electrons and holes share the same k-vector, inferring at the same time that the wave function spreading of electrons and holes in $\mathrm{k}$-space is low. Spatial quantum confinement of carriers in islands, however, according to Heisenberg's uncertainty relation, leads to a wave function spreading in the k-space which increases with decreasing island dimensions. We thus conclude that the studied $\mathrm{Si}_{0.7} \mathrm{Ge}_{0.3}$ islands, although revealing clear signatures of quantum confinement in the luminescence energies, provide only a comparably weak wave function spreading in k-space due to their spatial dimensions, which are large compared to quantum dots. Despite this, it is striking that, at $10 \mathrm{~K}$, the luminescence intensity of a single layer of $\mathrm{Si}_{0.7} \mathrm{Ge}_{0.3}$ islands is comparable to the intensity of the $\mathrm{Si}-\mathrm{TO}$ phonon replica from the whole volume of the excited Si matrix. This is even more remarkable as pure Ge quantum dots, which are considered to provide a no-phonon transition, ${ }^{21}$ show much weaker luminescence intensities with similar excitation power and at similar temperature. ${ }^{22-25}$

The observed enhanced luminescence intensity can be understood when considering the charge carrier probability densities predicted in the 3D band structure simulations of the islands. In Fig. 3(a), we show a cross-section of the 3D simulation displaying the band edges in growth direction of a $\mathrm{Si}_{0.7} \mathrm{Ge}_{0.3}$ island formed from nominally $40 \mathrm{ML}$ of deposited material. In addition, the ground state electron (dotted line) and hole (dashed-dotted line) probability densities in growth direction are plotted. The horizontal lines indicate the energy of the electron and hole ground state. As in pure Ge islands, the hole is strongly localized inside the
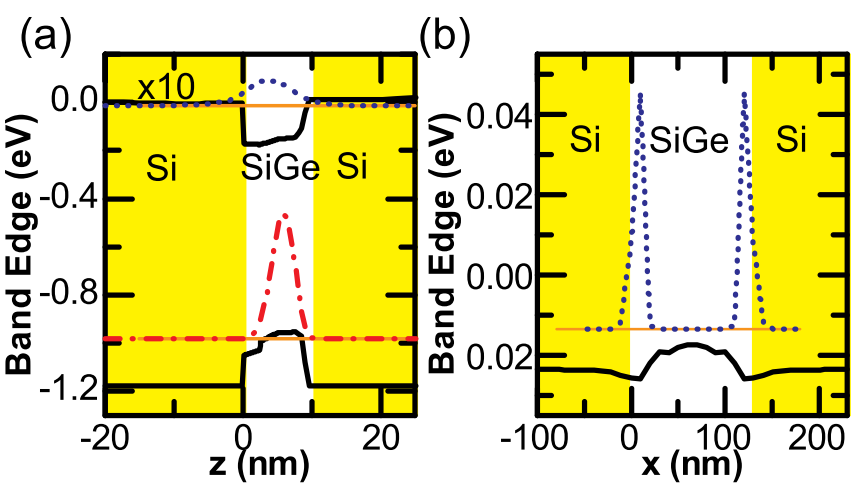

FIG. 3. (a) Band structure of a $\mathrm{Si}_{0.7} \mathrm{Ge}_{0.3}$ island with a thickness of nominally $40 \mathrm{ML}$ of deposited material seen in growth direction. The lower black solid line indicates the valence band. The upper black solid line indicates the conduction band multiplied by 10 for better visualization. The ground state energies of the electron and hole are given by a horizontal orange line. The electron (hole) probability density is shown by a blue dotted (red dashdotted) line. (b) Conduction band and electron probability of the same island as in (a) seen perpendicular to the growth direction.

island. ${ }^{22,26}$ Most remarkably, our simulations yield that the electron ground state is inside the island. This distinguishes our $\mathrm{Si}_{0.7} \mathrm{Ge}_{0.3}$ islands from nominally pure $\mathrm{Ge}$ islands where the electron ground state is located in the Si matrix above or below the island. ${ }^{14}$ Thus, we find a non-zero electron probability density over the whole height of the island. Fig. 3(b) shows a cross-section of the conduction band and the electron ground state probability density of the same island in the plane perpendicular to the growth direction through the apex of the island. Here, the electron is localized at the edges of the $\mathrm{Si}_{0.7} \mathrm{Ge}_{0.3}$ island. Thus, in addition to a type I confinement in z-direction, which can also be realized in quantum wells, ${ }^{12,13}$ the $\mathrm{Si}_{0.7} \mathrm{Ge}_{0.3}$ islands provide an in-plane confinement, preventing charge carrier diffusion. From the comparison of the PL data and the band structure simulations, we conclude that this 3D spatial type I confinement, which induces a large electron-hole wave function overlap in real space, is responsible for the comparably large luminescence intensity of the presented $\mathrm{Si}_{0.7} \mathrm{Ge}_{0.3}$ island ensembles. Furthermore, concerning optical transition probabilities, our results show that an optimization of the real space confinement properties of the nanostructures can overcompensate a low wave function spreading in $\mathrm{k}$-space.

In summary, we demonstrated the growth of laterally self-ordered $\mathrm{Si}_{0.7} \mathrm{Ge}_{0.3}$ islands by $\mathrm{Si}$ and $\mathrm{Ge}$ co-deposition. A systematic comparison of photoluminescence spectroscopy data and fit parameter-free 3D k $\cdot \mathrm{p}$ band structure simulations of island ensembles yielded enhanced optical properties compared to nominally pure $\mathrm{Ge}$ islands and $\mathrm{SiGe}$ quantum wells. This refers to both the ability for a processing-free optimization of the spatial confinement properties as well as for the lateral self-ordering, making SiGe islands, produced in a co-deposition approach, interesting nanostructures both for optical and electrical applications.

We gratefully acknowledge the financial support from the Nanosystems Initiative Munich (NIM) and of the Technische Universität München Institute for Advanced Study funded by the German Excellence Initiative and the International graduate school of science and engineering. 
${ }^{1}$ I. Kaminow, J. Lightwave Technol. 26, 994 (2008).

${ }^{2}$ L. Pavesi, Adv. Opt. Technol. 2008, 1.

${ }^{3}$ K. Tanabe, K. Watanabe, and Y. Arakawa, Sci. Rep. 2, 349 (2012).

${ }^{4}$ A. W. Fang, H. Park, O. Cohen, R. Jones, M. J. Paniccia, and J. E. Bowers, Opt. Express 14, 9203 (2006).

${ }^{5}$ B. Zheng, J. Michel, F. Y. G. Ren, L. C. Kimerling, D. C. Jacobson, and J. M. Poate, Appl. Phys. Lett. 64, 2842 (1994).

${ }^{6}$ M. V. Wolkin, J. Jorne, P. M. Fauchet, G. Allan, and C. Delerue, Phys. Rev. Lett. 82, 197 (1999).

${ }^{7}$ K. Murayama, H. Komatsu, S. Miyazaki, and M. Hirose, J. Lumin. 66-67, 319 (1995).

${ }^{8}$ K. Brunner, Rep. Prog. Phys. 65, 27 (2002).

${ }^{9}$ J. C. Sturm, H. Manoharan, L. C. Lenchyshyn, M. L. W. Thewalt, N. L. Rowell, J.-P. Noël, and D. C. Houghton, Phys. Rev. Lett. 66, 1362 (1991).

${ }^{10}$ L. Tsybeskov and D. Lockwood, Proc. IEEE 97, 1284 (2009).

${ }^{11}$ J. Brunner, P. Schittenhelm, J. Gondermann, B. Spangenberg, B. Hadam, T. Köster, H. Roskos, H. Kurz, H. Gossner, I. Eisele, and G. Abstreiter, J. Cryst. Growth 150, 1060 (1995).

${ }^{12}$ C. G. Van de Walle and R. M. Martin, Phys. Rev. B 34, 5621 (1986).

${ }^{13}$ R. People and J. C. Bean, Appl. Phys. Lett. 48, 538 (1986).

${ }^{14}$ M. Brehm, T. Suzuki, T. Fromherz, Z. Zhong, N. Hrauda, F. Hackl, J. Stangl, F. Schäffler, and G. Bauer, New J. Phys. 11, 063021 (2009).
${ }^{15}$ P. H. Tan, K. Brunner, D. Bougeard, and G. Abstreiter, Phys. Rev. B 68, 125302 (2003).

${ }^{16}$ A. Trellakis, T. Zibold, T. Andlauer, S. Birner, R. Smith, R. Morschl, and P. Vogl, J. Comput. Electron. 5, 285 (2006).

${ }^{17}$ G. Katsaros, P. Spathis, M. Stoffel, F. Fournel, M. Mongillo, F. Lefloch, A. Rastelli, O. Schmidt, and S. Franceschi, Nat. Nanotechnol. 5, 458 (2010).

${ }^{18}$ R. B. Hammond, T. C. McGill, and J. W. Mayer, Phys. Rev. B 13, 3566 (1976).

${ }^{19}$ R. Parsons and M. Thewalt, Solid State Commun. 21, 1087 (1977).

${ }^{20}$ R. Corkish and M. A. Green, J. Appl. Phys. 73, 3988 (1993).

${ }^{21}$ S. Fukatsu, H. Sunamura, Y. Shiraki, and S. Komiyama, Appl. Phys. Lett. 71, 258 (1997).

${ }^{22}$ M. Larsson, A. Elfving, P.-O. Holtz, G. Hansson, and W.-X. Ni, Physica E 16, 476 (2003).

${ }^{23}$ O. G. Schmidt, C. Lange, and K. Eberl, Appl. Phys. Lett. 75, 1905 (1999).

${ }^{24}$ M. Brehm, F. Montalenti, M. Grydlik, G. Vastola, H. Lichtenberger, N. Hrauda, M. J. Beck, T. Fromherz, F. Schäffler, L. Miglio, and G. Bauer, Phys. Rev. B 80, 205321 (2009).

${ }^{25}$ M. Larsson, A. Elfving, W. Ni, G. Hansson, and P. Holtz, Phys. Rev. B 73, 195319 (2006).

${ }^{26}$ J. J. Zhang, F. Montalenti, A. Rastelli, N. Hrauda, D. Scopece, H. Groiss, J. Stangl, F. Pezzoli, F. Schäffler, O. G. Schmidt, L. Miglio, and G. Bauer, Phys. Rev. Lett. 105, 166102 (2010). 